\title{
Predicting Malaria Transmission Risk in Endemic Areas of Iran: A Multilevel Modeling Using Climate and Socioeconomic Indicators
}

\author{
Khodadad Sheikhzadeh, ${ }^{1}$ Ali Akbar Haghdoost, ${ }^{1, *}$ Abbas Bahrampour, ${ }^{1,2}$ Ahmad Raeisi, ${ }^{3}$ Farzaneh \\ Zolala, ${ }^{1}$ Farshad Farzadfar, ${ }^{4}$ Amir Kasaeian, ${ }^{5}$ and Mahboubeh Parsaeian ${ }^{4,6}$ \\ ${ }^{1}$ Modeling in Health Research Center, Institute for Futures Studies in Health, Kerman University of Medical Sciences, Kerman, Iran \\ ${ }^{2}$ Department of Biostatistics and Epidemiology, Faculty of Health, Kerman University of Medical Sciences, Kerman, Iran \\ ${ }^{3}$ Department of Medical Entomology and Vector Control, School of Public Health, Tehran University of Medical Sciences, Tehran, Iran \\ ${ }^{4}$ Non-Communicable Diseases Research Center, Endocrinology and Metabolism Population Sciences Institute, Tehran University of Medical Sciences, Tehran, Iran \\ ${ }^{5}$ Hematology-Oncology and Stem Cell Transplantation Research Center, Tehran University of Medical Sciences, Tehran, Iran \\ ${ }^{6}$ Department of Epidemiology and Biostatistics, School of Public Health, Tehran University of Medical Sciences, Tehran, Iran \\ "Corresponding author: Ali Akbar Haghdoost, Kerman University of Medical Sciences, Medical University Campus, Haft-Bagh Highway, Kerman, Iran. Tel: +98-3431325820, Fax: \\ +98-3431325822, E-mail: ahaghdoost@gmail.com
}

Received 2016 December 28; Revised 2017 January 14; Accepted 2017 February 05.

\begin{abstract}
Background: Over the past years, the malaria elimination program has considerably decreased the malaria indigenous cases and confined their incidence to the southeastern areas of Iran encompassing 28 less developed counties with favorable transmission conditions.

Objectives: The aim of the study was to investigate the effects of climatic and socioeconomic indicators on malaria transmission and to predict malaria transmission risk until 2025 for all the southeastern endemic counties in Iran.

Methods: The aggregated data from indigenous malaria cases, as well as, climatic and socioeconomic indicators of malaria endemic areas were collected on the monthly basis from each county between 2005 and 2015. The collected variables were, then, estimated until 2025 using time series analysis. Considering the nature of these data, two different multilevel models were implemented for vivax and falciparum based on the socioeconomic variable that was used. Finally, applying two different models, two sets of estimates were obtained for malaria transmission risk in each county.

Results: The annual decline of malaria transmission was estimated to be $17 \%$ based on model 1, and $25 \%$ based on the model 2 for $\operatorname{vivax}(\mathrm{P}<0.001)$. These estimates were $13 \%$ and $21 \%$ for falciparum $(\mathrm{P}<0.001)$, respectively. For every increased unit in the wealth index, malaria transmission for vivax and falciparum decreased by $33 \%(\mathrm{P}=0.001)$ and $12 \%(\mathrm{P}=0.54)$, respectively. Also, for every increase in the mean years of schooling, the transmission decreased by $65 \%(\mathrm{P}<0.001)$ and $57 \%(\mathrm{P}=0.001)$ for vivax and falciparum. Conclusions: The results of this study, using climatic variables along with socioeconomic variables, indicated the obvious influence of socioeconomic status improvement on decreasing malaria transmission. According to the results, malaria transmission risk will considerably diminish in the next few years. The pattern of malaria transmission decline was consistent with the declining trend of malaria incidence which will move from the west to the east and from the north to the south in the years to come. The transmission risk for falciparum was considerably lower than that of vivax and the endemic areas of falciparum move towards 0 faster than vivax.
\end{abstract}

Keywords: Malaria, Iran, Multilevel Analysis, Geographic Information Systems, Risk

\section{Background}

Malaria has still remained to be a major health issue worldwide. According to the world health organization, there were an estimated 214 million malaria cases and 438 thousand malaria deaths in 2015 with the majority of the cases found in African countries (1). In spite of the extent and the severity of the problem in such areas, malaria cases have declined rapidly in recent years in a number of countries around the world including Iran. In the distant past, malaria was a major health issue in $\operatorname{Iran}(2,3)$. However, following the elimination program started in 2010, there has been an accelerated decline in malaria cases in Iran, and now they are limited only to some southeastern areas of the country $(4,5)$. According to the reports of the National Malaria Surveillance System, 799 malaria cases have been reported in Iran in 2015 from which 167 cases were locally transmitted and 632 were imported malaria cases.

Roughly about $90 \%$ of the locally-transmitted malaria cases occur across the southeastern endemic areas each year (6). The imported malaria cases, as well, enter these areas illegally via the eastern Iranian borders and are usually detected within the mentioned southeastern endemic areas. These areas encompass 28 counties which have always been the ground zero of malaria incidence in the past few years, owing to favorable weather conditions, abundance of malaria vectors, low socioeconomic conditions, prox- 
imity to foreign borders, and indiscriminate cross-border traffic. By focusing on the southeastern areas as the hub for malaria transmission and incidence, malaria elimination program in Iran has been scheduled to stop the local transmission of falciparum cases until 2018, vivax cases until 2022, and eventually, to obtain the certification of malaria elimination by 2025 (4).

Many factors are associated with malaria transmission including climatic and socioeconomic factors (7). Different epidemiological models have been employed, aiming at the investigation of the effects of these factors in malaria incidence, as well as predicting transmission risk (probability of malaria occurrence). The effects of climatic variables on malaria transmission are well known and these variables have been widely used in many studies regarding malaria transmission $(8,9)$. The socioeconomic factors are, also, among the significant variables associated with malaria transmission $(10,11)$ and have been used by some studies $(12,13)$. A fewer number of studies have used a combination of climatic variables along with socioeconomic variables for the estimation of malaria transmission $(14,15)$. Studies which were conducted in Iran have investigated the effects of a number of the above-mentioned variables (mostly climatic variables rather than socioeconomic factors) mainly in a limited geographical area (16, 17).

Studying both the climatic and socioeconomic variables can explain the trends of malaria cases and predict their future path more accurately. Considering the declining trend of local malaria transmission in Iran, especially in the southeastern areas, the prediction of local malaria transmission across Iranian endemic areas, while taking into account the determining factors (including both climatic and socioeconomic factors) on transmission, in the years ahead and in the time-place continuum, seems to be necessary and will act as an instrument for a better decision-making in the hands of the national executive directors of the elimination program. The maps illustrating the predicted transmission risk across different counties of the endemic areas in the years ahead can facilitate the localization of the malaria foci to concentrate all the preventive measures on these areas. In the conducted review, no studies were found to have carried out a comprehensive simultaneous study of both climatic and socioeconomic variables and the prediction of malaria transmission risk across all Iranian endemic areas.

In this study, we attempted to investigate the effects of climatic indicators alongside socioeconomic indicators on the malaria transmission across the endemic areas of Iran as one of the countries moving towards malaria elimination. Furthermore, using multi-level models, malaria transmission risk was predicted for the years to come. Our results can be, also, employed by other countries in a similar stage of elimination.

\section{Methods}

\subsection{Area of the Study}

This study was focused on an area encompassing 28 counties located in three southeastern provinces of Iran, covering nearly $283,000 \mathrm{~km}^{2}$ with a population of 4,640,000 people. The area neighbored Pakistan and Afghanistan towards the east, and the Persian Gulf and the Gulf of Oman towards the south (Figure 1). This area hosted a population with a low socioeconomic status and, also, provided more favorable conditions for malaria transmission (like the abundance of vectors, appropriate climatic conditions, and indiscriminate cross-border traffic).

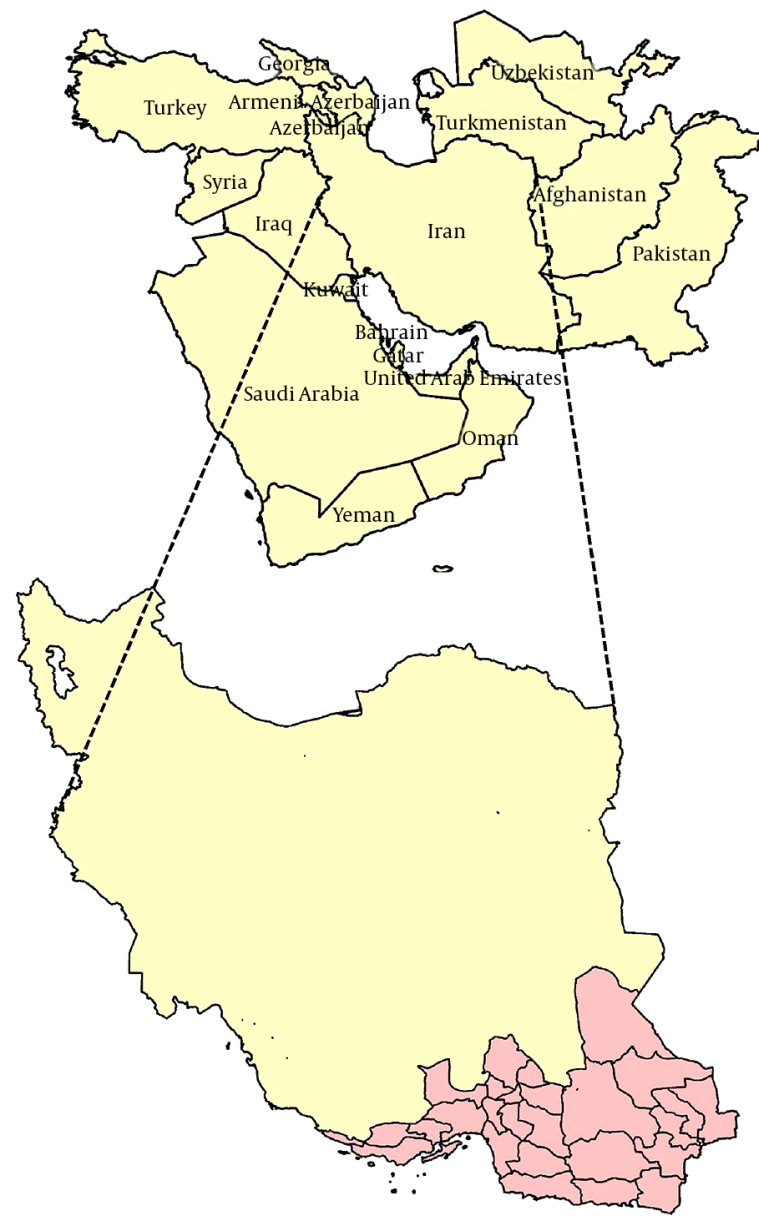

Figure 1. The Counties in the Southeast of Iran with Local Malaria Transmission on Which the Study Was Conducted 


\subsection{Data Collection and Management}

The study was a secondary analysis of the registered cases in the Malaria National Surveillance System which is based on the registration and the treatment of all malaria confirmed cases. Because of the extensive activities related to malaria control in all regions, particularly in malaria endemic areas of Iran in recent decades, the system is highly sensitive to case detection, registration, and data management which have been further improved after the malaria elimination program was launched $(18,19)$.

The registered data from confirmed indigenous cases (44,600 cases) since 2005 to 2015 separated by individual health centers across the endemic region were collected and cross-checked by two individuals to decrease any potential errors. Subsequently, the collected data were crosschecked with the data from the database of the Iranian ministry of health and medical education, as well as, that of the medical universities in the region. In addition, to ensure the accuracy of the recorded data, the authors sought the consultation of local malaria experts. The data pertaining to the health center of each county were, then, integrated into their respective county.

The required variables for predicting malaria transmission risk were selected based on the epidemiological aspects of malaria transmission. The monthly climatic data of the endemic region for the specific time period were collected from the Iranian meteorological organization separately by the synoptic centers. The employed socioeconomic indicators were wealth and education (years of schooling). These data were extracted from the indicators of the statistical center of Iran and the data from the censuses for the time period 2005 to 2015 and were performed on necessary manipulations. More details on the data extraction are available elsewhere (20).

\subsection{Data Analysis}

Data analysis initially comprised a step-by-step estimation of all the independent variables (Table 1) since 2015 until 2025 using time series analysis. In counties with no synoptic center, the climatic data were estimated based on the adjacent counties using co-kriging method via ArcGIS 9.3(ESRI, Redlands, CA).

The monthly incidence of locally transmitted vivax and falciparum malaria were our dependent variables. In order to estimate the monthly probability of malaria transmission, the mentioned variables were first converted to a zero-one variable, such that "one" would represent the presence of locally transmitted malaria in a month, and "zero" would represent the absence of such transmission. The hierarchical structure of the data called for a multilevel analysis in which months were the micro level and nested into the counties that were macro level. Using multilevel logistic analysis, the model was once implemented for vivax and once again for falciparum malaria. The bivariate models were performed first and subsequent to determining significant independent variables, the multivariable models were implemented. Due to the lack of overall fitness of the model at simultaneous use of the independent variables of education and wealth, each of these variables was used in a separate model. We were, eventually, left with four models: two models for vivax parasite (the independent variables for model 1 were year, mean of monthly temperature, monthly precipitation, monthly humidity, monthly highest temperature, and education, and the independent variables for model 2 were year, mean of monthly temperature, monthly precipitation, monthly humidity, monthly highest temperature, and wealth); and an additional two models for the falciparum parasite with the identical combination of the mentioned variables.

After determining the finalized model, the transmission risk estimation was carried out for all the given months using Stata software 11.0 (Stata Corp., College Station, TX). In the following step, the average transmission risk was calculated for different months and years, once for the entire endemic region, then over four time periods (2005 - 2009, 2010 - 2014, 2015 - 2019, 2020 - 2025) and, eventually, for six geographical zones within the endemic region. The geographical zones were: zone 1 encompassing northeastern counties of the endemic region; zone 2, the eastern counties neighboring Pakistan; zone 3, southern areas neighboring Pakistan and the Gulf of Oman; zone 4 including the central areas of the endemic region; zone 5 , northwestern areas; and zone 6 covering the southwestern cities, for the most part, neighboring the Persian Gulf (Please refer to Appendix 1 in the Supplementary File for the map of the categorized zone). The transmission risk in all the counties for vivax and falciparum parasites concerning the four time periods with 5-year intervals $(2005,2010$, $2015,2020,2025)$ were drawn and compared for all counties using ArcGIS 9.3.

\subsection{Ethical Considerations}

The collected data for the present study were aggregated and did not include personal information. The required permits for accessing these data had been obtained from the Iranian ministry of health and medical education.

\section{Results}

This study was conducted on the malaria aggregated data from 28 counties across the endemic region of Iran 
Table 1. Climatic and Socioeconomic Variables Which Were Used for the Estimation of Malaria Transmission Risk in Iranian Endemic Areas (2005 - 2025)

\begin{tabular}{|c|c|c|c|c|}
\hline Variable & Mean & Standard Deviation & Minimum & Maximum \\
\hline Mean of monthly temperature, ${ }^{\circ} \mathrm{C}$ & 25.5 & 7.3 & 2.4 & 38.7 \\
\hline Monthly highest temperature, ${ }^{\circ} \mathrm{C}$ & 36.0 & 7.2 & 17.4 & 51 \\
\hline Monthly precipitation, $\mathbf{m m}^{\mathrm{a}}$ & 9.8 & 17.5 & 0.0 & 208.2 \\
\hline Monthly humidity. \% & 42.3 & 16.9 & 9.4 & 85.0 \\
\hline Wealth & 0.3 & 0.9 & -1.9 & 2.8 \\
\hline Education (mean years of schooling) & 5.3 & 1.2 & 2.7 & 9.2 \\
\hline
\end{tabular}

in millimeter.

since 2005 to 2015 . The independent variables were climatic variables, year, wealth, and education (Table 1 ).

According to multilevel analysis, in model 1 and model 2, malaria transmission for vivax declined by $17 \%$ and $25 \%$ per year respectively which were significant $(\mathrm{P}<0.001)$. The similar figures, also, were significant for falciparum malaria at $13 \%$ and $21 \%$ for model 1 and 2, respectively (P $<0.001$ ). The annual transmission for falciparum malaria, in both models, showed a smaller compared compared to vivax malaria. For every year of increase in the mean years of schooling, malaria transmission for vivax and falciparum decreased by 65\% $(\mathrm{P}<0.001)$ and 57\% $(\mathrm{P}=0.001)$, respectively, which were statistically significant. Furthermore, for every increased unit in wealth indicator, malaria transmission for vivax and falciparum decreased by $33 \%$ ( $\mathrm{P}=$ $0.001)$ and $12 \%(P=0.54)$, respectively, which was only significant for vivax malaria. Based on the two models used, for every one-degree increase in the monthly mean temperature, transmission increased by $14 \%$ and $16 \%$ for vivax malaria $(\mathrm{P}<0.001)$, and by $25 \%$ and $26 \%$ for falciparum malaria $(\mathrm{P}<0.001)$, respectively (Table 2$)$.

Two different models were used based on the socioeconomic variable which was included in the model alongside the climatic variables; model-1, education variable was included; model-2, wealth variable was included.

Based on our estimation, the monthly average of malaria transmission risk across different months in the endemic areas showed that the highest malaria transmission risk for both vivax and falciparum was seen in the months of July and August, whereas the lowest malaria transmission risk was observed in Januarys and Decembers. Malaria transmission risk followed a more or less similar pattern across different months for vivax and falciparum. The only difference was that falciparum had more months with the lowest malaria transmission risk (Figure 2, time period 2005 - 2025).

Investigating the monthly average of malaria trans-
Figure 2. Monthly Average of Estimated Malaria Transmission Risk Using Climatic and Socioeconomic Variables for Vivax and Falciparum in Endemic Areas of Iran by Time Periods
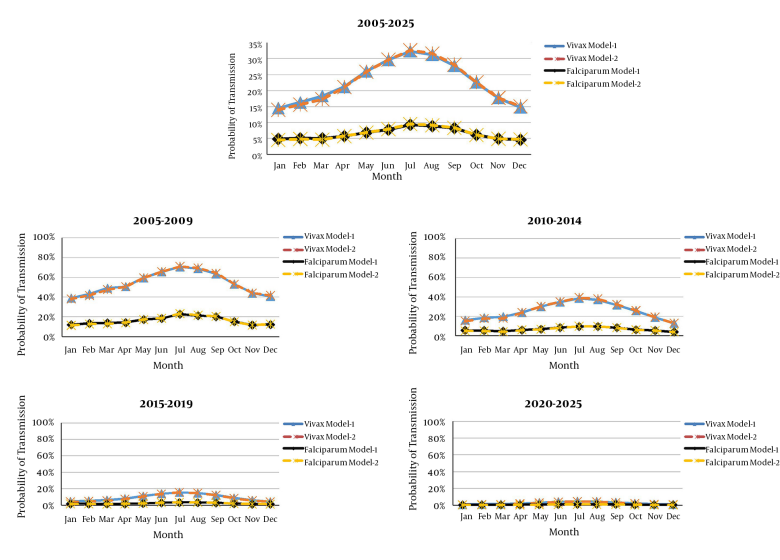

Based on the socioeconomic variable which was included in the model, alongside the climatic variables, two different models were used; model-1, education variable was included; model-2: wealth variable was included.

mission risk through different time periods since 2005 to 2025 showed that it gradually declined over time. The pattern for transmission risk across different months was roughly similar for vivax and falciparum. Maximum transmission risk in all four time periods was seen in July and August. The highest monthly transmission risk for vivax and falciparum malaria was observed in July during the time period "2005 to 2009" (Figure 2).

Investigating the designated zones showed that the risk of malaria transmission (monthly average) in all the zones was the highest in July and August. In addition, zone 3 held the highest transmission risk for vivax and falciparum malaria. In this zone, the malaria transmission risk was at its highest for June, July, August, and September, compared to the other months, a pattern not observ- 
Table 2. The Effects of Climatic and Socioeconomic Variables on Malaria Transmission Risk in Iranian Endemic Areas, 2005 - $2025^{\mathrm{a}}$

\begin{tabular}{|c|c|c|c|c|c|c|c|c|c|}
\hline \multirow[t]{2}{*}{ Model } & \multirow{3}{*}{$\begin{array}{c}\text { Variable } \\
\text { Year }\end{array}$} & \multicolumn{4}{|c|}{ Vivax } & \multicolumn{4}{|c|}{ Falciparum } \\
\hline & & \multirow{2}{*}{$\frac{\text { Odds ratio }}{0.83}$} & \multirow{2}{*}{$\frac{P}{<0.001}$} & \multicolumn{2}{|c|}{ 95\% Confidence interval } & \multirow{2}{*}{$\begin{array}{c}\text { Odds ratio } \\
0.87\end{array}$} & \multirow{2}{*}{$\frac{P}{<0.001}$} & \multicolumn{2}{|c|}{ 95\% Confidence interval } \\
\hline \multirow{6}{*}{ Model-1 } & & & & 0.80 & 0.87 & & & 0.81 & 0.94 \\
\hline & $\begin{array}{l}\text { Mean of monthly } \\
\text { temperature }\end{array}$ & 1.14 & $<0.001$ & 1.09 & 1.19 & 1.25 & $<0.001$ & 1.18 & 1.33 \\
\hline & $\begin{array}{l}\text { Monthly highest } \\
\text { temperature }\end{array}$ & 0.98 & 0.29 & 0.93 & 1.02 & 0.83 & $<0.001$ & 0.78 & 0.89 \\
\hline & Monthly precipitation & 1.001 & 0.55 & 0.997 & 1.005 & 0.99 & 0.07 & 0.98 & 1.00 \\
\hline & Monthly humidity & 1.02 & $<0.001$ & 1.01 & 1.03 & 1.003 & 0.37 & 0.99 & 1.01 \\
\hline & $\begin{array}{l}\text { Education (years of } \\
\text { schooling) }\end{array}$ & 0.35 & $<0.001$ & 0.28 & 0.43 & 0.43 & 0.001 & 0.26 & 0.70 \\
\hline \multirow{6}{*}{ Model-2 } & Year & 0.75 & $<0.001$ & 0.72 & 0.77 & 0.79 & $<0.001$ & 0.74 & 0.85 \\
\hline & $\begin{array}{l}\text { Mean of monthly } \\
\text { temperature }\end{array}$ & 1.16 & $<0.001$ & 1.12 & 1.22 & 1.26 & $<0.001$ & 1.19 & 1.34 \\
\hline & $\begin{array}{l}\text { Monthly highest } \\
\text { temperature }\end{array}$ & 0.95 & 0.03 & 0.91 & 0.99 & 0.83 & $<0.001$ & 0.78 & 0.88 \\
\hline & Monthly precipitation & 1.002 & 0.28 & 0.99 & 1.006 & 0.995 & 0.12 & 0.989 & 1.001 \\
\hline & Monthly humidity & 1.02 & $<0.001$ & 1.01 & 1.03 & 1.005 & 0.16 & 0.997 & 1.013 \\
\hline & Wealth & 0.67 & 0.001 & 0.53 & 0.84 & 0.88 & 0.54 & 0.59 & 1.31 \\
\hline
\end{tabular}

a All models were significant $(\mathrm{P}<0.001)$

able in the other zones. Zone 5 showed a lower transmission risk across different months compared to the other zones (Please refer to Appendix 2 in Supplementary File for monthly transmission risk in the categorized zone).

The developed GIS maps revealed that the transmission risk for vivax and falciparum in the endemic areas will gradually decline over the next few years based on the two adopted models used in five selected years. For all the selected years, falciparum transmission risk shows lower figures compared to that of vivax. Moreover, the direction of transmission risk decline is from the west towards the east and from the north to the south (Figure 3 ).

\section{Discussion}

The present study showed that malaria transmission risk in the endemic areas of Iran will decline over the next few years. Other than time, wealth and education were, also, associated with the decline of transmission. Malaria transmission risk was higher in July and August compared to the other months. Also, the direction of this decline in the years to come will be west to east and north to south. Southeastern areas of the endemic region (zone 3 ) had the highest transmission risk over time.

Malaria elimination program was initiated in Iran in 2010 and the interventions of this program have significantly accelerated the declining trend of malaria cases which had commenced prior to the elimination program. This declining trend of incidence took a sharper pace for vivax rather than falciparum. Although we did not find any studies in Iran regarding the estimation of malaria

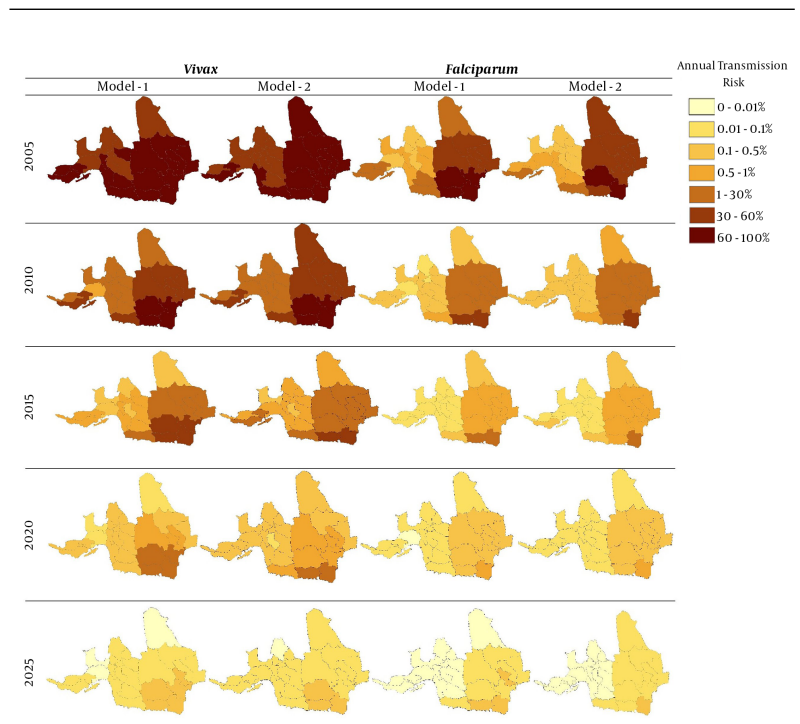

Figure 3. The Estimation of Annual Transmission Risk Using Two Different Multilevel Models (Based on the Socioeconomic Variable Which Was Included in the Model Alongside the Climatic Variables; Model-1, Education Variable Was Included; Model2, Wealth Variable Was Included) for Vivax and Falciparum in Endemic Areas of Iran by County and Year

transmission risk covering all of the endemic areas for several years, the declining trend of malaria transmission risk matched that of malaria incidence in the endemic region of Iran, which was supported by other studies $(21,22)$. The study results revealed that the degree of annual transmission decline, based on both models, was smaller for falciparum than for vivax which seems to reflect the lower de- 
clining speed of annual falciparum incidence in the region which was supported by another study conducted in the same areas (4).

Increase in the "years of schooling", as an important socioeconomic indicator, decreased malaria transmission. It seems that higher education brings about more appropriate behaviors regarding malaria prevention and treatment (23). Moreover, individuals with higher levels of education can be more educable and they understand the health concepts better (24). In addition, not only can higher education promise a better socioeconomic status in the future, but also it can be regarded as a proper indicator of the existing socioeconomic status.

In this study, a higher wealth indicator was associated with a lower malaria transmission across the endemic areas. Other studies have, also, approved the association between wealth and malaria incidence as well $(25,26)$. Even though malaria screening and treatment is totally free of charge in Iran and households do not make any payments for that, it seems that the domicile, the types of walls, coverings for doors and windows and where they are built within the structure, as well as, resting habits (like resting outdoors) are closely associated with wealth. These factors could affect the living conditions and as a result people who are exposed to bites experience greater risk of malaria transmission. Greater wealth and the higher level of education mean more access to health services, more learning capacity, and better living conditions. All these factors would limit the bite exposure and help people to more adapt to appropriate preventive measures and medical behaviors.

In this study, the risk for malaria transmission in the endemic areas was higher in July and August compared to other months of the year. The results from other studies on malaria outbreaks and incidence in Iran showed approximately a similar pattern of occurrence, which could support our finding $(27,28)$.

Although the conducted review did not come across any similar studies under conditions of low endemicity with vivax being responsible for the majority of the transmitted cases, it seems that vast deterioration of the falciparum cases over the past years and the minimization of the parasite reservoir have altered the transmission conditions. This may be why falciparum transmission (compared to vivax) was less influenced by increased wealth and education.

Recent years have observed a sharp decline in malaria incidence across Iranian endemic areas with vivax malaria as the dominant type of incidence. Our results, indicating the peak transmission risk during July and Augusts, gradual annual decrease of transmission risk, and higher transmission risk for vivax, were consistent with the actual current results recorded by the local malaria surveillance sys- tem which can, somehow, verify the accuracy of our estimations.

Among the categorized zones, transmission risk across different months was higher in zone 3 and lower in zone 5 , the latter of which encompasses more developed counties and currently boasts significantly lower incidence compared to the rest of the endemic areas. Whereas, zone 3 consists of less developed counties and due to vicinity to the border, is exposed to indiscriminate cross-border trafficking where malaria transmission conditions (including climatic conditions and abundance and the variety of vectors) are more favorable.

The review of the region's serial maps for vivax and falciparum parasites shows that the gradual trend of transmission risk declined during the years of the study and moved from the west to the east and from the north towards the south. It seems that over the next years, considering the measures of the elimination program, as well as, further development of the endemic areas, the transmission risk in the majority of these areas will be minimal leaving out only some limited less-developed areas adjacent to the eastern borders.

The present study estimated malaria transmission risk in the upcoming years for the different counties within the Iranian endemic areas using climatic variables alongside socioeconomic variables across a vast geographical span. These estimates specify interventional priorities of the Iranian malaria elimination program in the years to come. The application of entomological variables has always been important in the modeling for malaria transmission risk. With regard to the studied time period and geographical area, we could not use such variables in this study. If simultaneously used with other contributing factors for estimation of the transmission risk, incorporating entomological can result in a more accurate and credible risk estimation. Therefore, using entomological variable in the estimation of the malaria transmission risk is warranted in future studies.

\subsection{Conclusion}

Socioeconomic alongside climatic variables which were employed in this study are among the important contributing factors of malaria transmission. Having determined the trend and the direction of transmission risk decline across the counties within the endemic areas, this study pinpointed the areas requiring more attention in the future concerning preventive programs.

This study can act as a guide for countries with similar conditions to estimate malaria transmission risk for the future and apply more focus on the areas prone to malaria transmission. 


\section{Supplementary Material}

Supplementary material(s) is available here.

\section{Footnote}

Financial Disclosure: The authors declare that they have had no relevant financial interests or financial conflicts within the past 5 years and do not have for the foreseeable future.

\section{References}

1. Organization WH. . World Malaria Report 2015 [Internet]. 2015

2. Edrissian GH. Malaria in iran: Past and present situation. Iran J Parasitol. 2006;1(1):1-14.

3. Azizi MH, Bahadori M. Brief historical perspectives of malaria in Iran. Arch Iran Med. 2013;16(2):131-5. [PubMed: 23360639].

4. Sheikhzadeh K, Haghdoost AA, Bahrampour A, Zolala F, Raeisi A. Assessment of the impact of the malaria elimination programme on the burden of disease morbidity in endemic areas of Iran. Malar J. 2016;15:209. doi:10.1186/s12936-016-1267-9. [PubMed: 27074734].

5. Norouzinejad F, Ghaffari F, Raeisi A, Norouzinejad A. Epidemiological status of malaria in Iran, 2011-2014. Asian Pac J Trop Med. 2016;9(11):1055-61. doi: 10.1016/j.apjtm.2016.09.007. [PubMed: 27890364].

6. McKelvie WR, Haghdoost AA, Raeisi A. Defining and detecting malaria epidemics in south-east Iran. MalarJ. 2012;11:81. doi:10.1186/1475-287511-81. [PubMed: 22443235].

7. Loha E, Lindtjorn B. Predictors of Plasmodium falciparum malaria incidence in Chano Mille, South Ethiopia: a longitudinal study. Am JTrop Med Hyg. 2012;87(3):450-9. doi: 10.4269/ajtmh.2012.12-0155. [PubMed: 22826493].

8. Bi P, Tong S, Donald K, Parton KA, Ni J. Climatic variables and transmission of malaria: a 12-year data analysis in Shuchen County, China. Public Health Rep. 2003;118(1):65-71. doi:10.1016/S0033-3549(04)502182. [PubMed: 12604766].

9. Parham PE, Michael E. Modeling the effects of weather and climate change on malaria transmission. Environ Health Perspect. 2010;118(5):620-6. doi: 10.1289/ehp.0901256. [PubMed: 20435552].

10. Worrall E, Basu S, Hanson K. Is malaria a disease of poverty? A review of the literature. Trop Med Int Health. 2005;10(10):1047-59. doi: 10.1111/j.1365-3156.2005.01476.x. [PubMed:16185240].

11. Yadav K, Dhiman S, Rabha B, Saikia P, Veer V. Socio-economic determinants for malaria transmission risk in an endemic primary health centre in Assam, India. Infect Dis Poverty. 2014;3:19. doi: 10.1186/20499957-3-19. [PubMed: 24991410].

12. Banguero H. Socioeconomic factors associated with malaria in Colombia. Soc Sci Med. 1984;19(10):1099-104. doi: 10.1016/02779536(84)90313-7. [PubMed: 6523151].

13. Hagenlocher M, Castro MC. Mapping malaria risk and vulnerability in the United Republic of Tanzania: a spatial explicit model. Popul Health Metr. 2015;13(1):2. doi:10.1186/s12963-015-0036-2. [PubMed: 25674040].

14. Béguin A, Hales S, Rocklöv J, Åström C, Louis VR, Sauerborn R. The opposing effects of climate change and socio-economic development on the global distribution of malaria. Glob Environ Chang. 2011;21(4):1209-14. doi:10.1016/j.gloenvcha.2011.06.001.
15. van Lieshout $M$, Kovats RS, Livermore MTJ, Martens P. Climate change and malaria: analysis of the SRES climate and socioeconomic scenarios. Glob Environ Chang. 2004;14(1):87-99. doi: 10.1016/j.gloenvcha.2003.10.009.

16. Hanafi-Bojd AA, Vatandoost $\mathrm{H}$, Oshaghi MA, Charrahy Z, Haghdoost AA, Zamani G, et al. Spatial analysis and mapping of malaria risk in an endemic area, south of Iran: a GIS based decision making for planning of control. Acta Trop. 2012;122(1):132-7. doi: 10.1016/j.actatropica.2012.01.003. [PubMed: 22245147].

17. Ranjbar M, Shoghli A, Kolifarhood G, Tabatabaei SM, Amlashi M, Mohammadi M. Predicting factors for malaria re-introduction: an applied model in an elimination setting to prevent malaria outbreaks. Malar J. 2016;15:138. doi: 10.1186/s12936-016-1192-y. [PubMed: 26935846].

18. Akbari H, Majdzadeh R, Rahimi Foroushani A, Raeisi A. Timeliness of malaria surveillance system in iran. Iran J Public Health. 2013;42(1):3947. [PubMed: 23515191].

19. Raeisi A, Gouya MM, Nadim A, Ranjbar M, Hasanzehi A, Fallahnezhad M, et al. Determination of Malaria Epidemiological Status in Iran's Malarious Areas as Baseline Information for Implementation of Malaria Elimination Program in Iran. Iran J Public Health. 2013;42(3):326-33. [PubMed: 23641411].

20. Hashemi-Meshkini A, Kebriaeezadeh A, Jamshidi H, Akbari-Sari A, Rezaei-Darzi E, Mehdipour P, et al. Wealth-related Inequality in Utilization of Antihypertensive Medicines in Iran: an Ecological Study on Population Level Data. Arch Iran Med. 2016;19(2):116-22. [PubMed: 26838082].

21. Barati M, Keshavarz-valian H, Habibi-nokhandan M, Raeisi A, Faraji L, Salahi-moghaddam A. Spatial outline of malaria transmission in Iran. Asian Pac J Trop Med. 2012;5(10):789-95. doi: 10.1016/S19957645(12)60145-X. [PubMed: 23043918].

22. Hemami MR, Sari AA, Raeisi A, Vatandoost H, Majdzadeh R. Malaria elimination in Iran, importance and challenges. Int J Prev Med. 2013;4(1):88-94. [PubMed: 23413116].

23. Dike N, Onwujekwe O, Ojukwu J, Ikeme A, Uzochukwu B, Shu E. Influence of education and knowledge on perceptions and practices to control malaria in Southeast Nigeria. Soc Sci Med. 2006;63(1):103-6. doi: 10.1016/j.socscimed.2005.11.061. [PubMed: 16448735].

24. Galobardes B, Shaw M, Lawlor DA, Lynch JW, Davey Smith G. Indicators of socioeconomic position (part 1).J Epidemiol Community Health. 2006;60(1):7-12. doi:10.1136/jech.2004.023531. [PubMed: 16361448].

25. Thang ND, Erhart A, Speybroeck N, Hung le X, Thuan le K, Hung CT, et al. Malaria in central Vietnam: analysis of risk factors by multivariate analysis and classification tree models. Malar J. 2008;7:28. doi: 10.1186/1475-2875-7-28. [PubMed: 18234102].

26. Tusting LS, Willey B, Lucas H, Thompson J, Kafy HT, Smith R, et al. Socioeconomic development as an intervention against malaria: a systematic review and meta-analysis. Lancet. 2013;382(9896):963-72. doi: 10.1016/S0140-6736(13)60851-X. [PubMed: 23790353].

27. Ostovar A, Raeisi A, Haghdoost AA, Ranjbar M, Rahimi A, Sheikhzadeh $\mathrm{K}$, et al. Lessons learnt from malaria epidemics in the Islamic Republic of Iran. East Mediterr Health J. 2012;18(8):864-9. [PubMed: 23057376].

28. Moemenbellah-Fard MD, Saleh V, Banafshi O, Dabaghmanesh T. Malaria elimination trend from a hypo-endemic unstable active focus in southern Iran: predisposing climatic factors. Pathog Glob Health. 2012;106(6):358-65. doi: 10.1179/2047773212Y.0000000049. [PubMed: 23182141]. 